\title{
INTEGRO-DIFFERENTIAL EQUATIONS*
}

BY I. A. BARNETT

1. Introduction. It is proposed in this note to prove that if, in the integro-differential equation

$$
\frac{\partial \varphi(x, t)}{\partial t}=\int_{0}^{1} \varkappa(x, y) \varphi(y, t) d y,
$$

the real and continuous kernel $\varkappa(x, y)$ is also symmetric or skew-symmetric then there can be no solutions of the form

$$
e^{\lambda t} \sum_{k=0}^{p} \alpha_{k}(x) t^{k}
$$

unless all $\alpha_{k}(x), k \geqq 1$ are identically zero. $t$ It will then be pointed out that a similar property holds for systems of linear differential equations

$$
\frac{d \varphi_{i}}{d t}=\sum_{j=1}^{n} \varkappa_{i j} \varphi_{j},
$$

viz., if the $x_{i j}$ are real and constant (with respect to $t$ ), then in case the coefficient system is either symmetric or skew-symmetric, no real set of solutions can contain polynomials in $t$ as factors. This fact seems, to the best of the writer's knowledge, to have escaped attention in the literature. $\ddagger$

* Presented to the Society, December 29, 1923.

$\dagger$ See I. A. Barnett, Integro-differential equations with constant limits of integration, this BuLLETIN, vol. 26, pp. 193-203.

$\$$ I have recently had the privilege of examining, through the courtesy of Professor Brand of the University of Cincinnati, a course of lectures on linear differential equations that Professor Bôcher gave, in which the result for the symmetric case is given without proof. Professor Bôcher states there that Weierstrass worked out this case in 1858, tell years before he introduced elementary divisors. The writer has examined the collected works of Weierstrass but he was unable to find this result. 
2. Kernel Symmetric. Consider first the integro-differential equation (2) where $\varkappa(x, y)=\varkappa(y, x)$. Suppose there were a solution of the form (2). Substituting (2) in (1), we find

$$
\begin{aligned}
\sum_{k=0}^{p-1} t_{k} \int_{0}^{* 1} x(x, y) \alpha_{k}(y) d y+t^{p} \int_{0}^{* 1} x(x, y) \alpha_{p}(y) d y \\
=\sum_{k=0}^{p-1}(k+1) \alpha_{k+1}(x) t^{k}+\sum_{k=0}^{p-1} \lambda \alpha_{k}(x) t^{k}+\lambda \alpha_{p}(x) t^{p} .
\end{aligned}
$$

Writing $\mu=1 / \lambda$ one sees that this is equivalent to the following simultaneous system of integral equations

$$
\left\{\begin{aligned}
& \alpha_{p}(x)=\mu \int_{0}^{1} x(x, y) \alpha_{p}(y) d y, \\
& \alpha_{k}(x)=\mu \int_{0}^{\cdot 1} x(x, y) \alpha_{k}(y) d y-\mu(k+1) \alpha_{k+1}(x), \\
&(k-1, p-2, \ldots, 2,1,0) .
\end{aligned}\right.
$$

Suppose the integral equation

$$
{ }^{\prime} \iota_{p}(x)=\mu \int_{0}^{\cdot 1} \varkappa(x, y) \alpha_{p}(y) d y
$$

has a characteristic number $\mu_{1}$ of index $q_{1}$ with the corresponding characteristic functions $\boldsymbol{\Phi}_{1}^{(p)}, \boldsymbol{\Phi}_{2}^{(p)}, \cdots, \boldsymbol{\Phi}_{q_{1}}^{(p)}$ which may be supposed normed and orthogonalized. Then the solution $\alpha_{p}(x)$ is expressible as a linear combination of these solutions. Consider now the second equation in (4),

$$
\alpha_{p-1}(x)=\mu \int_{0}^{1} x(x, y) \alpha_{p-1}(y) d y-\mu_{p} \alpha_{p}(x),
$$

for the same characteristic number $\mu_{1}$. Necessary conditions that this last non-homogeneous integral equation have solutions are that all the associated characteristic functions of the kernel $x(x, y)$ be orthogonal to the function $\alpha_{p}(x)$. But, since the kernel is, by hypothesis, symmetric, it follows that the set of associated characteristic functions is the same as the set of characteristic functions of $\%$; viz., the set $\boldsymbol{\Phi}_{1}^{(p)}, \cdots, \boldsymbol{\Phi}_{q_{1}}^{(p)}$. This is impossible unless $\alpha_{p}(x)=0$ since $\int_{0}^{1} \boldsymbol{\Phi}_{i}^{(p)}(x) \boldsymbol{\Phi}_{i}^{(p)}(x) d x \neq 0, i=1,2, \cdots, q_{1}$. Reasoning in the same way, one can prove that $\alpha_{p-1} \equiv \ldots=\alpha_{1}=0$. 
3. Kernel Skew-Symmetric. Suppose now that the kernel $\varkappa(x, y)$ is skew-symmetric, i. e., $*(x, y)=-\varkappa(y, x)$. Equations (4) also hold in this case. It is well known in this case that the characteristic numbers are all of the form $\lambda i$ and that if $\left(\lambda_{1} i, \Phi_{1}(x)\right)$ are a characteristic number and function, respectively, of the kernel $\varkappa(x, y)$ then $\bar{\Phi}_{i}(x)$ is the associated characteristic function for the same characteristic number $\lambda_{1} i$ where $\bar{\Phi}_{1}(x)$ is the conjugate of $\boldsymbol{\Phi}_{1}(x)$. Hence, necessary conditions for the existence of a solution of equation (5) would be $\int_{0}^{1} \alpha_{p}(x) \Phi_{j}(x) d x=0, j=1, \cdots, q_{1}$. If then the $q_{1}$ characteristic functions $\boldsymbol{\Phi}_{1}^{(p)}, \ldots, \boldsymbol{\Phi}_{q_{1}}^{(p)}$ and the $q_{1}$ associated characteristic functions $\boldsymbol{\Phi}_{1}^{(p)}, \ldots, \boldsymbol{\Phi}_{q_{1}}^{(p)}$ are supposed normed and biorthogonalized, it is clear that not all of these necessary conditions can be satisfied since at least one of them reduces to $\int_{0}^{\prime} \Phi_{j}^{(p)}(x) \Phi_{j}^{(p)}(x) d x$ which equals unity.

4. Analogy for Differential Equations. Consider now the system of differential equations

$$
\frac{d \varphi_{i}}{d t}=\sum_{j=1}^{n} x_{i j} \varphi_{j},
$$

and a set of solutions $\left\{\varphi_{1}(t), \cdots, \varphi_{n}(t)\right\}$. It is well known that if $\left\{\psi_{1}(t), \cdots, \psi_{n}(t)\right\}$ is any set of solutions of the system of differential equations adjoint to (3), one has the relation

$$
\varphi_{1}(t) \psi_{1}(t)+\cdots+\varphi_{n}(t) \psi_{n}(t):=\text { constant. }
$$

But in case the coefficient system $\varkappa_{i j}$ is skew-symmetric, the set $\left\{\psi_{i}(t)\right\}$ coincides with the set $\left\{\varphi_{i}(t)\right\}^{*}$ and consequently one must have $\varphi_{1}^{2}(t)+\cdots+\varphi_{n}^{2}(t)=$ const. In other words the set $\left\{\varphi_{i}(t)\right\}$ cannot contain polynomials in $t$ as factors. In the symmetric case a solution of a given system with $t$ replaced by $-t$ is a solution of the adjoint system and so again we have the same result. $f$

The University of Cincinnati

* Goursat-Hedrick, Mathematical Analysis, vol. 2, part. II, p. 157.

$\dagger$ The writer is indebted to Professor 0. D. Kellogg for suggesting this proof by the method of adjoint systems. 